\title{
Multi-slice CT coronary angiography assessment of remodeling index in patients with low- to intermediate-risk stable angina
}

\author{
Shaimaa A. Mostafa 1* (D), Tarek Aboelazem¹, Osama Sanad' ${ }^{1}$, Haytham Abdelghafar and Ahmed Azam³
}

\begin{abstract}
Background: Early identification of vulnerable plaques by remodeling index prior to rupture and development of acute event is of considerable importance especially by a reliable non-invasive method as CT coronary angiography (CTA), so we aim to evaluate coronary artery remodeling index in patients with low- to intermediate-risk stable angina by CTA.

Results: This single-center, cross-sectional, observational study included 150 patients with stable angina with normal resting ECG, negative markers, normal systolic function by $2 \mathrm{D}$ echocardiography (EF $>50 \%$ ), and without regional wall motion abnormality at rest who were referred to MSCT evaluation of the coronary artery tree; the mean age was $56.8 \pm 6.4$ years, $83.3 \%$ had one-vessel disease, and $16.7 \%$ had two-vessel diseases. The mean remodeling index (RI) was $1.04 \pm 0.28,38 \%$ had significant positive remodeling, LAD was the most affected vessel (55.3), and proximal lesions were predominant in 48.5\%; there was a statistically significant positive correlation between RI and cholesterol, triglyceride, LDL, duration of DM, HBA1c, and plaque burden $(P<0.001)$ and a statistically significant negative correlation with $\mathrm{HDL}(P<0.001)$. Predictors of higher RI were positive family history, diabetes mellitus, low HDL, HBA1c, and plaque burden\% $(P<0.001)$. Patients with remodeling index $>1.1$ were diabetic, hypertensive, smoker, with longer duration of diabetes mellitus, higher HBA1C, cholesterol, triglyceride, $L D L$, plaque burden, wall lumen ratio, stenosis area, and lower HDL.
\end{abstract}

Conclusion: CTA was able to detect the presence and extent of early, non-obstructive but significant coronary artery-positive remodeling in patients with low- to intermediate-risk stable angina patients.

Trial registration: NCT03963609, 22 May 2019

Keywords: CT coronary angiography, Remodeling index, Stable angina

\section{Background}

The response of coronary arteries to atherosclerosis and plaque growth manifested as either compensatory enlargement or shrinkage which is known as coronary artery remodeling. Increase in vessel size due to the outward expansion of the vessel wall is called positive remodeling, while a decrease in vessel size due to vessel shrinkage is defined as negative remodeling [1].

The assessment of positive remodeling of coronary arteries has attracted a lot of attention due to the

\footnotetext{
* Correspondence: shaimaamustafa2011@gmail.com

${ }^{1}$ Cardiovascular Department, Faculty of Medicine, Benha Univerisity Hospital, Banha, Egypt

Full list of author information is available at the end of the article
}

association of positively remodeled coronary plaques with plaque vulnerability and the propensity to cause future cardiac events based on histopathological and clinical studies [2].

Remodeling index is defined as the ratio of the maximum vessel area (or diameter) to a normal reference vessel area (or diameter), and plaques are classified as having significant positive remodeling when the RI is $>1.1$ [3].

Although invasive coronary angiography is considered the gold standard for the diagnosis of coronary artery disease since it provides an excellent visualization of coronary lumen change, coronary angiography can only provide a two-dimensional outline of the coronary lumen and cannot demonstrate the complex nature of 
atherosclerotic plaques which are responsible for the association between the angiographic findings and clinical outcome [4].

Although IVUS is the standard reference for the assessment of coronary plaque composition and progression in clinical studies, it is an invasive procedure which is not commonly performed in routine clinical practice [4].

CTA has been widely used as an effective less invasive imaging modality to diagnose CAD as it allows the evaluation of coronary plaque characteristics and classification of coronary plaque compositions which has important clinical implications and association with myocardial ischemia [5].

It has been reported that almost two thirds of acute coronary events occur in noncritical lesions with less than $50 \%$ lumen stenosis, highlighting the necessity to detect and analyze these plaques at early stages, especially in low-risk patients, which would improve risk stratification without the need for more invasive procedures

CT imaging will be most useful in patients with an intermediate likelihood of CAS. In patients with low pretest likelihood, the false-positive rate may be too high, and in patients with high pretest likelihood, sensitivity may not be sufficiently high. Meijboom et al. have recently presented a careful analysis of the diagnostic value of coronary CTA, stratified according to the pretest likelihood of disease. They also found that the technique is most useful in patients with a low to intermediate likelihood of CAS [6].

\section{Aim}

The aim was to evaluate coronary artery remodeling index in patients with low- to intermediate-risk stable angina by multi-slice computed tomography (MSCT) coronary angiography.

\section{Patients and methods Study design}

The study design is a single-center, cross-sectional, observational study conducted in the period from January 2016 to January 2018; the study was approved by the local ethical committee and all patients signed informed consent.

All patients were subjected to thorough history taking, complete general and local examination, laboratory investigation, resting ECG, echocardiography, and then MSCT coronary angiography.

\section{Inclusion and exclusion criteria Inclusion criteria}

The study included adult patients, $>18$ years of both genders with first attack, low- to intermediate-risk stable angina with normal resting ECG, negative markers, and normal systolic function by 2D echocardiography $(E F>50 \%)$ and without regional wall motion abnormality at rest who were referred to MSCT evaluation of the coronary artery tree.

\section{Exclusion criteria}

We excluded patients with a previous acute coronary syndrome or revascularization, those with renal impairment or dye hypersensitivity, patients with morbid obesity (BMI $\left.>40 \mathrm{~kg} / \mathrm{m}^{2}\right)$, rhythm other than sinus rhythm, and inability to hold breath for $10 \mathrm{~s}$ to acquire the image and lesions with heavy calcium score.

\section{Echocardiography}

All patients were examined in the left lateral position using (PHILIPS, EPIC 7C) machine with multi-frequency transducer. Standard views for two dimensional, M mode, Doppler, and tissue Doppler studies were obtained according to the recommendation of the American Society of Echocardiography for the assessment of left ventricular end-systolic and diastolic function.

\section{MSCT coronary angiography}

The study was carried out on Aquilion Prime 320 slices Toshiba Medical System Corporation-Nishinasuno, Tokyo, Japan. The reassurance of the patients from the entrance to the scanning room was performed, including an appropriate knowledge of the whole process. A stable venous line should be available, this requiring an 18 - to 20 -gauge needle placed into an antecubital vein. The preparation for the study must include a pre-exam testing of the ability of the patient for sustaining a breathhold long enough for the purposes of the examination. All patients received beta-blocking agent (ivabradine 5 $\mathrm{mg}$ once or twice if needed) to decrease heart rate to about $65-70$ beats per minute.

Patients were placed within the gantry of the CT scanner in the supine position. According to the expected location of the coronary arteries obtained from the AP and lateral scout images, a preliminary scan without contrast injection was performed to determine the total calcium burden (calcium score) of the coronary tree; followed by ECG gated acquisition for coronary angiography with contrast material injected via a pump with total volume about $65-75 \mathrm{cc} /$ patient. In all cases, the administration of the contrast was performed at a rate of $4-5 \mathrm{cc} / \mathrm{s}$ in order to more reliably enhance the vascular bed.

\section{Post-processing}

The acquired images were transferred for post-processing on Vitrea WS-Vital Images, Minnetonka, Minnesota, USA. The most expansive atherosclerotic lesion in the original MDCT data sets was identified; serial multiplanar reconstructions (slice thickness $1 \mathrm{~mm}$ ) were 
rendered in an orientation perpendicular to the longitudinal axis of the respective coronary artery segment. The cross-sectional vessel area was determined in a reference segment without detectable plaque proximal to and as close as possible to the respective coronary lesion (in absence of a segment without plaque, the least diseased segment between the lesion and the coronary ostium or major bifurcations), and several measurements were acquired including the lumen area, vessel wall surface area, and wall thickness, as well as wall percent area (wall thickness/vessel surface area) and wall/lumen percentage. Also, these measurements were repeated at the site of maximum arterial remodeling and compared with the reference segment measurements.

\section{Statistical methods}

Data management and statistical analysis were done using SPSS vs. 25. Numerical data was summarized as means and standard deviations or median and ranges. Categorical data was summarized as numbers and percentages. Comparisons between patients with remodeling index (RI) less than or equal to 1.1 and patients with RI above 1.1 were done using independent $t$ test or Mann-Whitney $U$ test for normally and non-normally distributed numerical variables respectively. Categorical variables were compared using the chi-square test. Correlations were done using the Pearson or Spearman correlation if appropriate. " $r$ " is the correlation coefficient. It ranges from -1 to +1 ; -1 indicates a strong negative correlation, +1 indicates a strong positive correlation, and 0 indicates no correlation. Linear regression analysis was done for the remodeling index as a dependent variable. All coefficients with 95\% confidence intervals were calculated. All $P$ values were two-sided. $P$ values less than 0.05 were considered significant

\section{Results}

During the period from January 2016 to January 2018,225 patients with first attack stable angina were referred to MSCT coronary angiography, and only 150 patients were included for the assessment of the remodeling index.

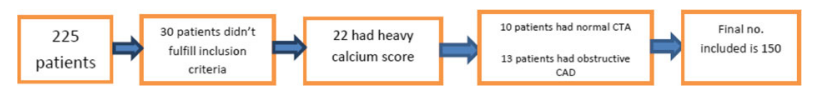

\section{Demographics and baseline criteria of the study population}

The mean age of the study population was $56.8 \pm$ 6.4 years; $87.3 \%$ were male, $42.7 \%$ were hypertensive, $63.3 \%$ were smokers, and $55.3 \%$ were diabetic with a mean duration of diabetes (years) of $13.37 \pm 4.49$, and
43.3\% had a family history of premature CAD. None of the patients was on statin therapy.

Mean serum cholesterol was $212.6 \pm 33.8 \mathrm{mg} / \mathrm{dl}$, mean TG was $213 \pm 37.8 \mathrm{mg} / \mathrm{dl}$, mean LDL was $97.5 \pm 19.5 \mathrm{mg} / \mathrm{dl}$, HDL was $42 \pm 7.9 \mathrm{mg} / \mathrm{dl}$, and mean HBA1c was $7 \pm 2.6 \%$.

By echocardiography, the mean EF\% was $60.9 \pm$ $5.5 \% .27 .3 \%$ of the study patients were referred before MSCT coronary angiography for a stress test (exercise ECG or stress echocardiography) that was not conclusive for stress-induced myocardial ischemia, but for the remaining patients, MSCT coronary angiography was the first choice.

\section{MSCT coronary angiography}

The number of diseased vessels

83.3\% had one-vessel disease and $16.7 \%$ had two-vessel disease; none of the patients had three-vessel disease or left main disease.

The LAD vessel was affected in $55.3 \%$ ( $44 \%$ as a single vessel and $11.3 \%$ as two-vessel disease), LCX was affected in $21.3 \%$, and RCA was affected in $18.7 \%$ while the first diagonal and obtuse marginal were affected in $3.3 \%$ and $1.4 \%$ respectively.

Proximal lesions were predominant in $48.5 \%$, then mid-segment lesions in $33.1 \%$, and distal lesions in $6.8 \%$.

\section{Assessment of the affected segments}

The mean remodeling index was $1.04 \pm 0.28,38 \%$ had significant positive remodeling ( $\mathrm{RI}>1.1$ ), mean plaque burden (\%) was $53.3 \pm 6.8$, mean wall lumen ratio (\%) was $147.1 \pm 34.5$, mean stenosis area (\%) was $39.5 \pm 16.7$, stenosis diameter (\%) was $24.2 \pm 11.9$, and length $(\mathrm{mm})$ was $18.5 \pm 10.6$ (Fig. 1).

\section{Correlation analysis for remodeling index}

There was a statistically significant positive correlation between remodeling index and cholesterol, triglyceride, LDL, duration of DM, HBA1c, and plaque burden $(P<0.001)$ and a statistically significant negative correlation with HDL $(P<0.001)$ (Table 1$)$.

\section{Predictors for high remodeling index}

Multivariate linear regression analysis was done for prediction of remodeling index. Variance inflation factor (VIF) was used to assess collinearity. VIF of all factors were below 5 , indicating no multicollinearity problems in the model.

By linear regression analysis; positive family history, diabetes mellitus, low HDL, HBA1c, and plaque burden\% were predictors of high remodeling index (Table 2). 


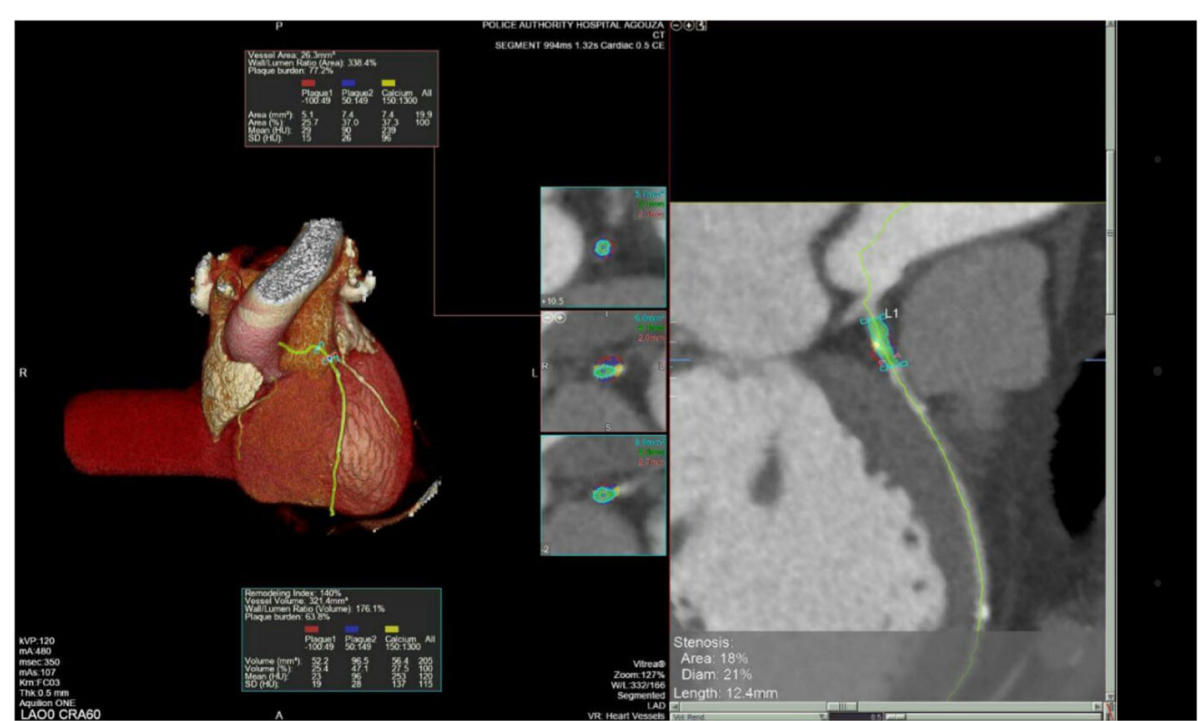

Fig. 1 MSCT angiography coronary of a male patient, a 42-year-old smoker, and complaining of chest pain: HbA1c was 11, cholesterol level was 249, TGS 250, HDL 35, and LDL 100. CT angiography shows the proximal LAD lesion with remodeling index 1.4\%, plaque burden $77.2 \%$, wall lumen ratio $338.4 \%$, stenosis area $18 \%$, stenosis diameter $21 \%$, and stenosis length $12.4 \mathrm{~mm}$

\section{Comparison between study population with remodeling index $\leq 1.1$ and $>1.1$}

Thirty-eight percent of the patients had remodeling index $>1.1$, and when compared to those with RI $<1.1$, they were found to have statistically higher prevalence of diabetes $(100 \%$ vs. $28 \%, P<0.001)$, hypertension $(63.2 \%$ vs. $30.1 \%, P<0.001)$, and smoking ( $86 \%$ vs. $49.5 \%, P<0.001)$ and longer duration of diabetes $(14.41 \pm 4.07$ vs. $11.08 \pm$ 4.59 $P=0.001)$.

Also, patients with remodeling index $>1.1$ had statistically higher HBA1c, serum cholesterol, triglyceride, and LDL $(P<0.001)$ and lower HDL $(P<0.001)$.

By MSCT coronary angiography, there was higher plaque burden, wall lumen ratio, and stenotic area in patients with remodeling index $>1.1$ (Table 3 ).

\section{Discussion}

Coronary artery disease is defined as clinically significant when luminal narrowing is present, typically at the $50 \%$ diameter reduction threshold. However, in early atherosclerosis, the first arterial changes consisted of compensatory enlargement of both the outer wall of the vessel as well as the lumen, termed compensatory enlargement or positive remodeling [7].

Positive remodeling had the best sensitivity and specificity ( $87 \%$ and $88 \%$, respectively) as compared to low attenuation and spotty calcification to identify vulnerable plaque [8]. Being less conditional to image noise as plaque attenuation and having a more quantitative definition as the Napkin-ring sign, positive remodeling might become a more robust marker for vulnerable plaques [9].
Recent studies have demonstrated the incremental prognostic value of adverse plaque features over luminal stenosis. Comprehensive coronary plaque assessment holds the potential to significantly improve individual risk assessment [9].

The Promise trial found also that high-risk plaque was especially apparent among subjects with nonobstructive CAD on CTA (adjusted hazard ratio 4.31 vs. 2.64) among this low-intermediate-risk cohort (33\% with 10 -year atherosclerotic cardiovascular disease risk < 7.5\%) [10].

So, the aim of the present study was the evaluation of coronary artery remodeling index in patients with lowto intermediate-risk stable angina by MSCT coronary angiography.

In the present study, 150 patients with coronary artery remolding fulfilled the criteria and were subjected to thorough history taking, laboratory investigation, ECG, resting echocardiography, and MSCT coronary angiography for the evaluation of the degree of remodeling index and its predictors and correlations.

83.3\% had one-vessel disease and $16.7 \%$ had two-vessel diseases; none of the patients had three-vessel or left main disease. LAD was the most affected vessel followed by LCX and RCA, and the least affected were diagonal and obtuse marginal. Proximal lesions were predominant as well as mid and distal lesions.

The mean remodeling index was $1.04 \pm 0.28$. There was a statistically significant positive correlation between remodeling index and cholesterol, triglyceride, LDL, duration of DM, HBA1c, and plaque burden $(P<0.001)$ and a statistically significant negative correlation with $\mathrm{HDL}$ $(P<0.001)$. By linear regression analysis, positive family 
Table 1 Correlation analysis for remodeling index (RI)

\begin{tabular}{|c|c|c|}
\hline & & $\mathrm{Rl}$ \\
\hline \multirow[t]{2}{*}{ Age (years) } & $r$ & -0.055 \\
\hline & $P$ value & 0.505 \\
\hline \multirow[t]{2}{*}{ Duration of DM } & $r$ & 0.518 \\
\hline & $P$ value & $<0.001$ \\
\hline \multirow[t]{2}{*}{$\mathrm{HbA} 1 \mathrm{c}$} & $r$ & 0.663 \\
\hline & $P$ value & $<0.001$ \\
\hline \multirow[t]{2}{*}{$\mathrm{HDL}$} & $r$ & -0.689 \\
\hline & $P$ value & $<0.001$ \\
\hline \multirow[t]{2}{*}{ TGS } & $r$ & 0.654 \\
\hline & $P$ value & $<0.001$ \\
\hline \multirow[t]{2}{*}{ LDL } & $r$ & 0.524 \\
\hline & $P$ value & $<0.001$ \\
\hline \multirow[t]{2}{*}{ Cholesterol } & $r$ & 0.652 \\
\hline & $P$ value & $<0.001$ \\
\hline \multirow[t]{2}{*}{ Wall lumen ratio (\%) } & $r$ & -0.182 \\
\hline & $P$ value & 0.026 \\
\hline \multirow[t]{2}{*}{ Stenosis area (\%) } & $r$ & -0.16 \\
\hline & $P$ value & 0.051 \\
\hline \multirow[t]{2}{*}{ Stenosis diameter (\%) } & r & -0.081 \\
\hline & $P$ value & 0.325 \\
\hline \multirow[t]{2}{*}{ Length } & $r$ & -0.08 \\
\hline & $P$ value & 0.33 \\
\hline \multirow[t]{2}{*}{ Plaque burden (\%) } & $r$ & 0.531 \\
\hline & $P$ value & $<0.001$ \\
\hline \multirow[t]{2}{*}{ LVED (\%) } & $r$ & -0.131 \\
\hline & $P$ value & 0.11 \\
\hline
\end{tabular}

history, diabetes mellitus, low HDL, HBA1c, and plaque burden $\%$ were predictors of high remodeling index

Patients with remodeling index $>1.1$ were diabetic, hypertensive, smoker, had longer duration of diabetes mellitus, higher HBA1c, cholesterol, triglyceride, LDL, plaque burden, wall lumen ratio, stenotic area, and had lower HDL $(P<0.001)$.

The finding of this study highlights two facts: the first is the careful consideration of symptoms even in patients with low to intermediate stable angina (normal ECG, good systolic function, no segmental wall motion abnormalities, negative markers of myocardial ischemia) and the second fact is the importance of correction of risk factors especially diabetes and dyslipidemia regarding the significant correlation with the remodeling index besides being predictors of positive remodeling.

The selection of the appropriate test to patients with low- to intermediate-risk stable angina is a matter of debate; in the present study, $27.3 \%$ of the patients were referred before MSCT coronary angiography for stress test (exercise ECG or stress echocardiography) that was nonconclusive for stress-induced myocardial ischemia, but for the remaining patients, MSCT coronary angiography was the first choice.

In 2016, the National Institute for Health and Care Excellence (NICE), the organization that guides healthcare in the UK, updated its chest pain guideline and made coronary CTA as the first test for all patients without established CAD who present with typical or atypical angina or with non-anginal chest pain plus an abnormal resting electrocardiogram [11].

On the other hand, the current US stable ischemic heart disease guidelines favor noninvasive functional testing for myocardial ischemia in most patients, reserving anatomic testing using coronary computed tomography angiography

Table 2 Linear regression analysis for remodeling index (RI)

\begin{tabular}{|c|c|c|c|c|c|c|}
\hline & B & SE & Beta & $t$ & $95.0 \% \mathrm{Cl}$ for $B$ & $P$ value \\
\hline Constant & 0.422 & 0.175 & & 2.414 & (0.076 to 0.767$)$ & 0.017 \\
\hline Age & -0.001 & 0.002 & -0.022 & -0.577 & $(-0.004$ to 0.002$)$ & 0.565 \\
\hline Family history & 0.122 & 0.023 & 0.221 & 5.26 & (0.076 to 0.169$)$ & $<0.001$ \\
\hline HTN & 0.022 & 0.022 & 0.039 & 0.98 & $(-0.022$ to 0.066$)$ & 0.329 \\
\hline DM & 0.145 & 0.035 & 0.263 & 4.165 & (0.076 to 0.214$)$ & $<0.001$ \\
\hline Cholesterol & 0 & 0.001 & 0.049 & 0.629 & $(-0.001$ to 0.002$)$ & 0.53 \\
\hline Plaque burden (\%) & 0.011 & 0.002 & 0.278 & 6.655 & (0.008 to 0.015$)$ & $<0.001$ \\
\hline TGS & 0.001 & 0.001 & 0.141 & 1.901 & (0.0004 to 0.002$)$ & 0.059 \\
\hline LDL & -0.001 & 0.001 & -0.07 & -1.352 & $(-0.002$ to 0.0005$)$ & 0.179 \\
\hline $\mathrm{HDL}$ & -0.01 & 0.002 & -0.274 & -5.872 & $(-0.013$ to -0.006$)$ & $<0.001$ \\
\hline $\mathrm{HbA} 1 \mathrm{c}$ & 0.015 & 0.006 & 0.14 & 2.343 & (0.002 to 0.027$)$ & 0.021 \\
\hline Stenosis area (\%) & 0 & 0.001 & -0.011 & -0.279 & $(-0.001$ to 0.001$)$ & 0.781 \\
\hline Length & 0.001 & 0.001 & 0.04 & 1.069 & ( -0.001 to 0.003$)$ & 0.287 \\
\hline
\end{tabular}

$B$ regression coefficient, Beta standardized coefficient, SE standard error, 95\% Cl 95\% confidence interval 
Table 3 Comparison between variables with $\mathrm{RI} \leq 1.1$ and $>1.1$

\begin{tabular}{|c|c|c|c|c|c|c|}
\hline & & \multicolumn{2}{|c|}{$\mathrm{RI} \leq 1.1(n=93)$} & \multicolumn{2}{|c|}{$\mathrm{RI}>1.1(n=57)$} & \multirow[b]{2}{*}{$P$ value } \\
\hline & & Mean & $\pm S D$ & Mean & $\pm S D$ & \\
\hline \multirow[t]{5}{*}{ Risk factors } & Age (years) & 56.7 & 5.6 & 56.8 & 7.2 & 0.949 \\
\hline & Duration of DM (years) & 11.08 & 4.59 & 14.42 & 4.07 & 0.002 \\
\hline & DM & No. (\%) & $26(28)$ & No. (\%) & $57(100)$ & $<0.001$ \\
\hline & HTN & No. (\%) & $28(30.0)$ & No. (\%) & $36(63.0)$ & 0.001 \\
\hline & Smoking & No. (\%) & $46(49.5)$ & №. (\%) & $49(86.0)$ & $<0.001$ \\
\hline \multirow[t]{5}{*}{ Laboratory } & $\mathrm{HbA1c}$ & 5.8 & 2 & 9 & 2.3 & $<0.001$ \\
\hline & Cholesterol & 196.9 & 28.4 & 238.2 & 25.3 & $<0.001$ \\
\hline & TGS & 196.6 & 33.5 & 239.6 & 28 & $<0.001$ \\
\hline & LDL & 90.5 & 15.5 & 108.9 & 20.1 & $<0.001$ \\
\hline & $\mathrm{HDL}$ & 45.4 & 7.4 & 36.4 & 5 & $<0.001$ \\
\hline Echo & LVEF (\%) & 61.1 & 5.5 & 60.7 & 5.6 & 0.585 \\
\hline \multirow[t]{5}{*}{ MSCT CA } & Plaque burden (\%) & 51.2 & 6.2 & 56.6 & 6.3 & $<0.001$ \\
\hline & Wall lumen ratio (\%) & 153.9 & 35 & 136.1 & 31 & 0.002 \\
\hline & Stenosis area (\%) & \multicolumn{2}{|c|}{$37.0(7.0-76.0)$} & \multicolumn{2}{|c|}{$34(3-76)$} & 0.01 \\
\hline & Stenosis diameter (\%) & \multicolumn{2}{|c|}{$23(10-61)$} & \multicolumn{2}{|c|}{$21(70.0-68.0)$} & 0.348 \\
\hline & Length & \multicolumn{2}{|c|}{$15.5(9.4-92.4)$} & \multicolumn{2}{|c|}{$14.9(7.8-54.5)$} & 0.683 \\
\hline
\end{tabular}

(CTA) for patients without established CAD who have already undergone functional testing (inconclusive results or ongoing symptoms) or are unable to undergo functional testing [12].

The choice of noninvasive tests should always be individualized, accounting for local expertise, results of prior testing, and patient factors that influence test appropriateness and accuracy, but coronary CTA should at least always be an option available to patients and providers [13].

In the present study, proximal lesions were predominate which is an important finding regarding the substudy of the PROSPECT trial which demonstrated that vulnerable plaques are frequently seen in the proximal coronary tree, followed by the mid-coronary tree and the least in the distal coronary tree [14].

The data of correlation of remodeling index with demographic and risk factors were variable; Burke et al. [15] found that positive remodeling was more in men and older group but Abdeldayem et al. [7] found that no correlation was noted between either age, sex, and coronary risk factors with remodeling index, but the latter result can be explained by the small number of the study population ( 35 patients) and so less prevalence of risk factors.

On the other hand, Britten et al. [16] found that cardiovascular risk factors like hypertension and hypercholesterolemia were associated with reduced positive or even negative remodeling. Moreover, the total number of classical cardiovascular risk factors was a strong predictor for reduced positive remodeling $(P$ for trend $<0.001)$ meaning advanced stage of atherosclerosis from non-flow limiting to flow-limiting lesion as measured by fractional flow reserve.

We should consider that most acute coronary syndromes were initiated by sudden changes of mildly stenotic lesions, commonly found in positively remodeled arterial regions, rather than from progression of lesions already causing significant luminal narrowing; so, the identification of mildly stenotic but vulnerable atherosclerotic lesions and the overall plaque burden could provide better markers of coronary risk than measuring of luminal stenosis [7].

CT coronary angiography (CTA) has a sensitivity of $89 \%$ and specificity of $96 \%$ for the detection of presence, extent, angiographic severity, and composition of coronary atherosclerosis [17] even in early stage with positive remodeling which is difficult to be diagnosed by invasive coronary angiography which mainly examine the wall or by non-invasive test which unmask flow-limiting lesions. So, it enables to better identify patients most likely to benefit from aggressive preventive medications and lifestyle interventions.

\section{Conclusion}

CTA was able to detect the presence and extent of early, non- obstructive, but significant coronary artery positive remodeling in patients with low- to intermediate-risk stable angina patients.

\section{Limitation}

Besides being a single-center experience, a small number of patients also lack long-term follow-up to detect the 
outcome of patients with high positive remodeling or regression of the remodeling with control of risk factors.

\section{Abbreviations}

CAD: Coronary artery disease; CTA: CT coronary angiography; MSCT: Multislice computed tomography; RI: Remodeling index

\section{Acknowledgements}

Not applicable

\section{Authors' contributions}

SM carried out the protocol of the study which included the study design, selection criteria of the patients, share in the analysis of the data, and writing, reviewing, and publishing of the article. TA and OS shared in the writing, statistical analysis, and review process. HA and AA shared the analysis of the imaging of the patients and analysis of the MSCT and collecting the data. All authors read and approved the final manuscript.

\section{Funding}

Not applicable

\section{Availability of data and materials}

All materials are available.

\section{Ethics approval and consent to participate}

The study was approved by the local ethical committee (Benha University Hospital), and all patients signed informed consent to participate in the study.

\section{Consent for publication}

Not applicable

\section{Competing interests}

The authors declare that they have no competing interests

\section{Author details}

${ }^{1}$ Cardiovascular Department, Faculty of Medicine, Benha Univerisity Hospital, Banha, Egypt. ${ }^{2}$ Radiology Department, El-Agoza Police Hospital, Giza, Egypt.

${ }^{3}$ Cardiovascular Department, El-Agoza Police Hospital, Giza, Egypt.

Received: 27 May 2019 Accepted: 24 July 2019

Published online: 11 September 2019

\section{References}

1. Nishioka T, Luo H, Eigler N, Berglund H, Kim C, Siegel R (1996) Contribution of inadequate compensatory enlargement to development of human coronary artery stenosis: an in vivo intravascular ultrasound study. J Am Coll Cardiol 27:1571-1576

2. Pasterkamp G, Schoneveld A, van der Wal A et al (1998) Relation of arterial geometry to luminal narrowing and histologic markers for plaque vulnerability: the remodeling paradox. J Am Coll Cardiol 32:655-662

3. Giannopoulos AA, Benz DC, Gräni C, Buechel RR (2017) Imaging the event-prone coronary artery plaque. J Nucl Cardiol. 6:1-13

4. Topol E, Nissen S (1995) Our preoccupation with coronary luminology: the dissociation between clinical and angiographic findings in ischemic heart disease. Circulation. 92:2333-2342

5. Virmani R, Burke A, Farb A, Kolodgie F (2006) Pathology of the vulnerable plaque. J Am Coll Cardiol 47:C13-C18

6. Meijboom WB, van Mieghem CA, Mollet NR et al (2007) 64-slice computed tomography coronary angiography in patients with high, intermediate, or low pretest probability of significant coronary artery disease. J Am Coll Cardiol. 50:1469-1475

7. Abdeldayem E, Ibrahim A, Amr M, Ahmed E, Genedi S, Wahid H (2015) Positive remodeling index by MSCT coronary angiography: a prognostic factor for early detection of plaque rupture and vulnerability. Egypt J Radiol Nucl Med 46:13-22

8. Motoyama S, Sarai M, Narula J et al (2013) Coronary CT angiography and high-risk plaque morphology. Cardiovasc Interv Ther 28:1-8

9. Kolossváry M, Szilveszter B, Merkely B, Maurovich-Horvat P (2017) Plaque imaging with $\mathrm{C} T$ - a comprehensive review on coronary $\mathrm{CT}$ angiography based risk assessment. Cardiovasc Diagn Ther. 7(5):489-506
10. Ferencik M, Mayrhofer T, Bittner DO et al (2018) Use of high-risk coronary atherosclerotic plaque detection for risk stratification of patients with stable chest pain: A Secondary Analysis of the PROMISE Randomized Clinical Trial. JAMA Cardiol 3:144-152

11. National Institute for Health and Care Excellence (2010) Clinical guideline CG95 "Chest pain of recent onset: assessment and diagnosis.". NICE, London

12. Fihn SD, Gardin JM, Abrams J et al (2012) 2012 ACCF/AHA/ACP/AATS/ PCNA/SCAI/STS Guideline for the diagnosis and management of patients with stable ischemic heart disease: a report of the American College of Cardiology Foundation/American Heart Association Task Force on Practice Guidelines, and the American College of Physicians, American Association for Thoracic Surgery, Preventive Cardiovascular Nurses Association, Society for Cardiovascular Angiography and Interventions, and Society of Thoracic Surgeons. J Am Coll Cardiol 60:e44-e164

13. Todd C (2018) Coronary CTA should be the initial test in most patients with stable chest pain. JACC

14. Otsuka K, Fukuda S, Tanaka A et al (2013) Napkin-ring sign on coronary CT angiography for the prediction of acute coronary syndrome. JACC Cardiovasc Imaging 6:448-455

15. Burke A, Kolodgie F, Farb A, Weber D, Virmani R (2002) Morphological predictors of arterial remodeling in coronary atherosclerosis. Circulation 105:297-303

16. Britten MB, Zeiher AM (2003) Schächinger V Effects of cardiovascular risk factors on coronary artery remodeling in patients with mild atherosclerosis. Coron Artery Dis. 14(6):415-422

17. Miller JM, Rochitte CE, Dewey M et al (2008) Diagnostic performance of coronary angiography by 64-row CT. N Engl J Med. 359:2324-2336

\section{Publisher's Note}

Springer Nature remains neutral with regard to jurisdictional claims in published maps and institutional affiliations.

\section{Submit your manuscript to a SpringerOpen ${ }^{\circ}$ journal and benefit from:}

- Convenient online submission

- Rigorous peer review

- Open access: articles freely available online

High visibility within the field

- Retaining the copyright to your article

Submit your next manuscript at $\boldsymbol{\nabla}$ springeropen.com 